Article

\title{
Citric Acid Regulated Fabrication of Macroporous $\mathrm{TiO}_{2}$
}

\author{
Rui Chen, Ningning Shao, Xiaoquan Zhou and Tiehong Chen *
}

School of Materials Science and Engineering, Institute of New Catalytic Materials Science, Key Laboratory of Advanced Energy Materials Chemistry (MOE), Collaborative Innovation Center of Chemical Science and Engineering (Tianjin), Nankai University, Tianjin 300350, China; mse-chenr@nankai.edu.cn (R.C.); shaoningning0305@163.com (N.S.); zhouxq-nankai-2017@163.com (X.Z.)

* Correspondence: chenth@nankai.edu.cn

Received: 1 December 2019; Accepted: 17 January 2020; Published: 3 February 2020

check for updates

\begin{abstract}
Macroporous $\mathrm{TiO}_{2}$ monolith was prepared by a microphase separation method. After citric acid was added to the synthesis procedure, the yield of the titanium precursor has been significantly increased, and the stability of macroporous structure can be obviously enhanced. Anatase and rutile phase of $\mathrm{TiO}_{2}$ were obtained after a $550{ }^{\circ} \mathrm{C}$ and $800{ }^{\circ} \mathrm{C}$ calcination, respectively.
\end{abstract}

Keywords: titanium oxide; macroporous; citric acid; photocatalyst

\section{Introduction}

As one of the important inorganic materials, titanium oxide, has proven to be a safe, nontoxic chemical, and $\mathrm{TiO}_{2}$ has been widely used in catalysis, optical coatings, gas sensing, lithium ion batteries [1-7]. Also, much attention has been paid to $\mathrm{TiO}_{2}$ because of its highly efficient photocatalytic activity [8-11]. Owing to its good chemical and physical properties, $\mathrm{TiO}_{2}$ with different morphologies and structures, such as quantuam dots, microspheres, nanorods, nanowires, nanoplate, and hierarchical 3D-microstructures have been prepared through soluthermal, sol-gel, hard-template methods [12-22].

It is well known that catalysts with porous structures will provide high surface area, more active site, and easy for mass transfer. $\mathrm{TiO}_{2}$ with mesoporous, macroporous and hierarchical porous structures have been reported, $\mathrm{Wu}$. et al. reported a hard template method to fabricate a connected three-dimensional ordered macroporous (3DOM) materials, polystyrene (PS) colloid was used as a template in an ethanol/water mixed solution of citric acid [23]. The hard template method was further developed to synthesis a $\mathrm{TiO}_{2}$-carbon nanocomposite in a three dimensionally ordered macroporous framework with a pore-hierarchical structure [24].

In this paper, neither hard or soft templating agent was added in the synthesis procedure, nitrogen-doped titanium dioxide with microporous-macroporous multi-scale porous structure was prepared by a microphase separation method. Citric acid was used as a stabilizing agent to control the hydrolysis of butyl titanate, and the macroporous structure can be effectively stabilized. Compared with the procedure without citric acid, the macroporous morphology and structure of titanium precursor was greatly improved and the product are fully constructed by macrouprous structures. The amount of citric acid and other reaction conditions on the formation of macropores and crystalline phase were investigated. By changing the calcination temperature, crystalline anatase and rutile phases were obtained. The materials were characterized by XRD, SEM, TEM, $\mathrm{N}_{2}$ adsorption-desorption, XPS, UV-vis spectra, and photocatalytic activity. 


\section{Materials and Methods}

\subsection{Materials}

Citric acid was purchased from Tianjin Ruijinte Chemical Co., Ltd, Tianjin, China. Tetrabutyl titanate was purchased from Guangfu Fine Chemical Research Institute, Tianjin, China, Ammonium hydroxide and anhydrous ethanol were purchased from Tianjin Chemical Reagent No.3 Factory. Rhodamine B (RhB) was purchased from ACROS. All reagents are analytically pure and do not require further purification.

\subsection{Synthesis}

A mixed solution of anhydrous ethanol and deionized water were formulated with ethanol weight percent of 95\%. Different amount of citric acid was added to $40 \mathrm{~mL}$ of the above solution, and the mixture was stirred for $30 \mathrm{~min}$. After it was sufficiently dissolved, $10 \mathrm{~mL}$ of concentrated aqueous ammonia was slowly added dropwise to the mixture to form a white flocculent precipitate. After stirring well, the stirring speed was slowed down, and another $10 \mathrm{~mL}$ of concentrated ammonia water was added dropwise at a constant rate, and the precipitate gradually dissolved. Then, $6 \mathrm{~mL}$ of butyl titanate was added dropwise to the reaction system, the stirring was stopped, and the mixture stayed for another $12 \mathrm{~h}$. After centrifugation, the product was thoroughly washed with deionized water, absolute ethanol, and dried at $60{ }^{\circ} \mathrm{C}$ for $24 \mathrm{~h}$.

The obtained product was calcined at $350{ }^{\circ} \mathrm{C}, 550{ }^{\circ} \mathrm{C}$, and $800{ }^{\circ} \mathrm{C}$ for $4 \mathrm{~h}$, respectively.

\subsection{Characterization}

The crystal structure of the sample was characterized by Rigaku D/Max-2500 diffraction analyzer (San Jose, CA, USA), Cu K $\alpha 1\left(1.5406 \AA\right.$ ) radiation operated at $40 \mathrm{kV}, 10-70^{\circ}$, scanning speed $4^{\circ} / \mathrm{min}$. The morphology of the sample was observed by SHIMADZU SS-550 scanning electron microscope (Shimadzu Corporation, Kyoto, Japan) at $5 \mathrm{keV}$. The morphology, elemental distribution, and lattice information of the samples were obtained by field emission transmission electron microscopy (FE-TEM, JEM-2800, JEOL, Tokyo, Japan), attachments in transmission electron microscopy (Energy Dispersive Spectrdmeter). UV-vis spectrum was recorded on SHIMADZU UV-2450 (Shimadzu Corporation, Kyoto, Japan). $\mathrm{N}_{2}$ adsorption-desorption isotherms were measured on a BELSORP-mini surface analyzer (Mercer Instruments, Passy, France) at liquid nitrogen temperature $(77 \mathrm{~K})$. The samples were degassed at $250{ }^{\circ} \mathrm{C}$ for $4 \mathrm{~h}$ prior to the measurement. The surface area was obtained by the Brunauer-Emmett-Teller (BET) method, and pore size distribution was calculated from the adsorption branch of the isotherm by the Barret-Joyner-Halenda (BJH) model. The surface composition of the samples are determined by X-ray photoelectron spectroscopy (XPS, Thermo Scientific ESCALAB 250Xi, Thermofisher scientific, Waltham, MA, USA).

\subsection{Photocatalytic Performance}

Rhodamine $\mathrm{B}(\mathrm{RB})$ is a typical representative of organic dye contaminants in water [7]. It is used to detect the photocatalytic activity of $\mathrm{TiO}_{2}$ under ultraviolet light.

In a typical photocatalytic experiment, $20 \mathrm{mg}$ catalyst was well dispersed into $100 \mathrm{~mL} \mathrm{RB}$ $\left(10^{-5} \mathrm{M}\right)$ solution, no ultrasonic dispersion is used because the ultrasonic pre-treatment will destroy the macroporous structure of the catalyst. In the catalytic reactor, the cooling water was passed through the quartz tube to make sure the reaction will not be disturbed by the reaction temperature. Firstly, the RB solution with/without catalyst was magnetically stirred in the dark for $30 \mathrm{~min}$ to allow an adsorption/desorption equilibrium. Then, a $150 \mathrm{~W}$ mercury lamp (central wavelength of $325 \mathrm{~nm}$ ) was turned on and off every $20 \mathrm{~min}$. In each single stop, the supernatant was centrifuged $(8000 \mathrm{r} / \mathrm{min}$, $15 \mathrm{~min}$ ), and measured by an ultraviolet-visible spectrophotometer at $\lambda=552 \mathrm{~nm}$, using deionized water as reference. The concentration of the RB solution at a certain time can be determined from the calibration curve. The conversion rate of RB at each time was calculated as $X=\left(C_{0}-C\right) / C_{0}$. 
In order to determine the durability and stability of the photocatalyst, a recycle test for photodegradation was also carried out.

\section{Results and Discussion}

\subsection{Macroporous $\mathrm{TiO}_{2}$}

The shape and size of the obtained precursor were investigated by SEM observation. Figure 1 shows the SEM image of the precursor prepared through the microphase separation method, without the addition of citric acid in the raw material. It can be seen that the product is composed of lots of blocks in micrometer size with irregular shapes, and higher magnification picture shows that few of the blocks incompletely formed porous structure (Figure 1b), which shows that the precursor has a tendency to form three-dimensional macroporous pores in ethanol/water solution, but does not form a stable macroporous pore structure in a wide range. If $0.32 \mathrm{~g}$ of citric acid was added to the original mixture (see Figure 1c,d), the obtained product has a macroporous structure with high yield, the macropores are long and narrow, with macropores of 1-8 $\mu \mathrm{m}$. The macroprous monolith exhibits a cylindrical channel parallel to each other, perpendicular to the surface of the monolith. It can be seen that only one side of the parallel porous channel is open, the other end of the channel is closed.
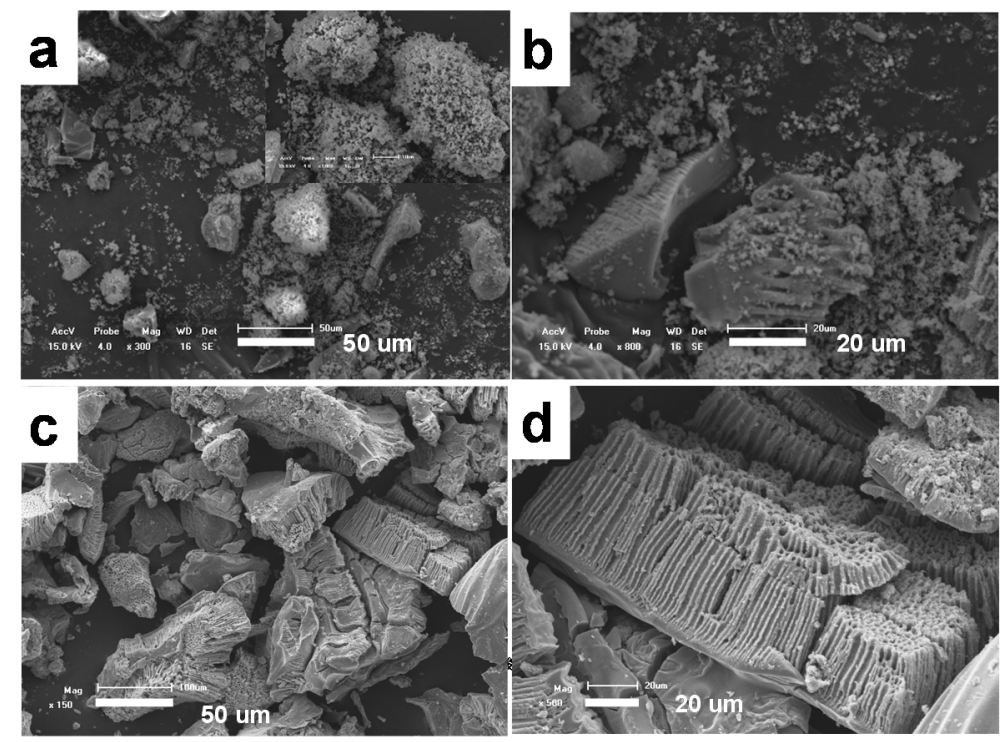

Figure 1. SEM images of titanium precursor prepared $(\mathbf{a}, \mathbf{b})$ without the addition of citric acid, $(\mathbf{c}, \mathbf{d})$ with 0.32 g citric acid.

The crystal structure of all samples was determined by powder X-ray diffraction. Figure 2 is an X-ray diffraction (XRD) pattern of the sample before calcination and after calcination at different temperatures. In the XRD pattern, the titanium precursor showed no diffraction peaks, indicating that the uncalcined titanium precursor was amorphous. If the precursor was calcined at $350{ }^{\circ} \mathrm{C}$ for $4 \mathrm{~h}$, a crystalline titanium oxide was obtained. As shown in Figure $3 \mathrm{~b}$, it is a typical anatase phase (JCPDS 21-1272), and the diffraction peak appeared broad, indicating poor crystallinity of the $\mathrm{TiO}_{2}-350$. The diffraction peak of the product obtained by calcination at $550{ }^{\circ} \mathrm{C}$ is still a typical anatase phase (JCPDS 21-1272), and the peak shape is sharper, indicating that the obtained product is a highly crystalline anatase phase. IF the precursor was further treated by a high temperature calcination, $800^{\circ} \mathrm{C}$ for $4 \mathrm{~h}$, the obtained product shows a highly crystalline rutile phase (JCPDS 34-0180). The crystallite size of the calcinated $\mathrm{TiO}_{2}$ samples were calculated, for $\mathrm{TiO}_{2}-350$, the size of the particle is calculated as $\mathrm{D}(101)=13 \mathrm{~nm}$, if the calcination temperature is $550^{\circ} \mathrm{C}$, the crystallite size is about $\mathrm{D}(101)=21 \mathrm{~nm}$. For $\mathrm{TiO}_{2}-800$, the average particles size is about $\mathrm{D}(110)=52 \mathrm{~nm}$. 


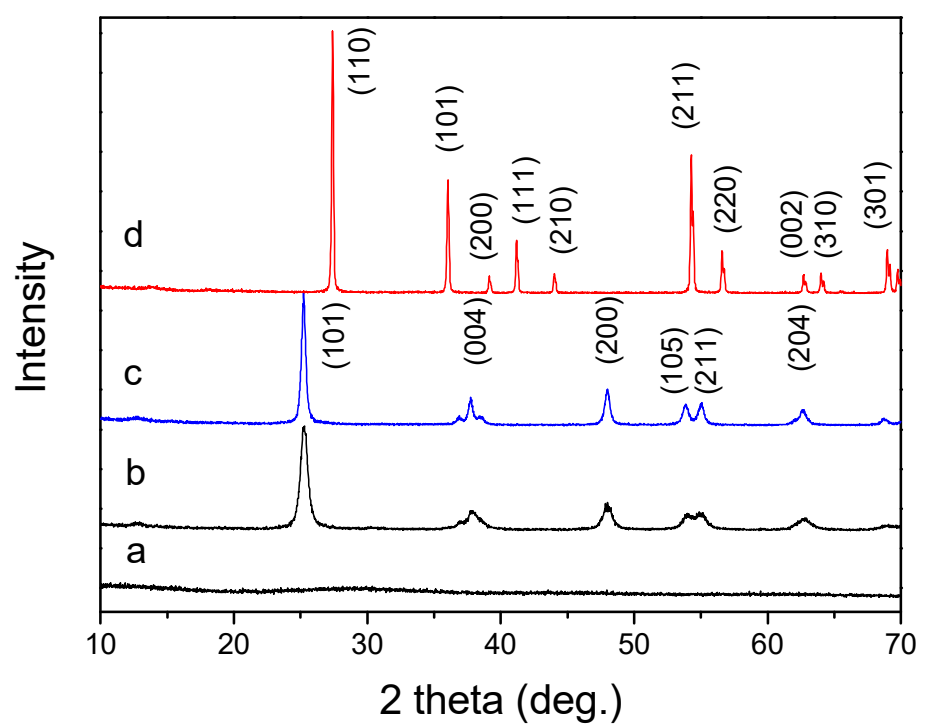

Figure 2. XRD patterns of titanium samples, (a) titanium precursor, (b) $\mathrm{TiO}_{2}-350$, (c) $\mathrm{TiO}_{2}-550$, (d) $\mathrm{TiO}_{2}-800$.

The morphology and microstructure of the calcined titanium oxide were further evidenced by SEM and TEM images (Figure 3). From the SEM images, it can be seen that the macroporous structure was well inherited, even after a high temperature $800^{\circ} \mathrm{C}$ calcination. High magnification SEM images show that the pore wall of the macropores was constructed by aggregations of nanoparticles.

The physico-chemical adsorption properties and pore parameters of the porous titania product were studied by $\mathrm{N}_{2}$ adsorption-desorption (Figure 4). Before calcination, titanium precursor has type I adsorption isotherm (Figure 4a), without a significant hysteresis loop, and the resulting product is presumed to contain micropores. The Brunaure-Emmett-Teller specific surface area of the precursor is $247 \mathrm{~m}^{2} / \mathrm{g}$, and the $\mathrm{BJH}$ pore size distribution showed an average pore diameter of $1.7 \mathrm{~nm}$, which belongs to ultramicropores (1-2 nm). Therefore, it is speculated that the macroporous material contains micropores and mesopores, having a relatively wide pore size distribution. Since no templating agent is used in the synthesis process, it is speculated that the obtained micropores and mesopores are irregular pores formed by the accumulation of amorphous titanium dioxide particles or the gaps between the particles, and the pore size of the pores should be relatively wide.

The type of adsorption isotherm (see Figure $4 \mathrm{~b}$ ) of the sample calcined at $550{ }^{\circ} \mathrm{C}$ can still be attributed to the Langmuir Type I adsorption isotherm, with a hysteresis loop. It is speculated that during the crystallization of anatase, the growth of titanium dioxide crystals causes the microporous/mesoporous structure formed by the particles to be deformed and partially damaged. The BET specific surface area was reduced to $48.8 \mathrm{~m}^{2} / \mathrm{g}$. The hysteresis ring appears at a relative pressure of about 0.4 , indicating the presence of mesopores, and the $\mathrm{BJH}$ pore size distribution in the mesoporous region has a wide range, and the average $\mathrm{BJH}$ pore diameter is $4.6 \mathrm{~nm}$.

After calcination at $800{ }^{\circ} \mathrm{C}$, the isotherm (see Figure 4c) is completely incompatible with the standard classification of IUPAC adsorption isotherms. The hysteresis loop in the region with higher relative pressure may be the structure of its macropores and mesopores, there is no more micropores contained in $\mathrm{TiO}_{2}-800$. The BET specific surface area of the product after calcination at $800{ }^{\circ} \mathrm{C}$ was suddenly reduced to $17 \mathrm{~m}^{2} / \mathrm{g}$, which also confirmed that the microporous in the product had disappeared after calcination at a high temperature of $800{ }^{\circ} \mathrm{C}$. BJH pore size distribution in the mesoporous region has a wide range from 3-10 $\mathrm{nm}$. We can speculate that in the high-temperature crystal transformation process, the macro- and mesopores formed by the particle accumulation are retained, micropores were completely destroyed due to the growth of the crystal particles. 

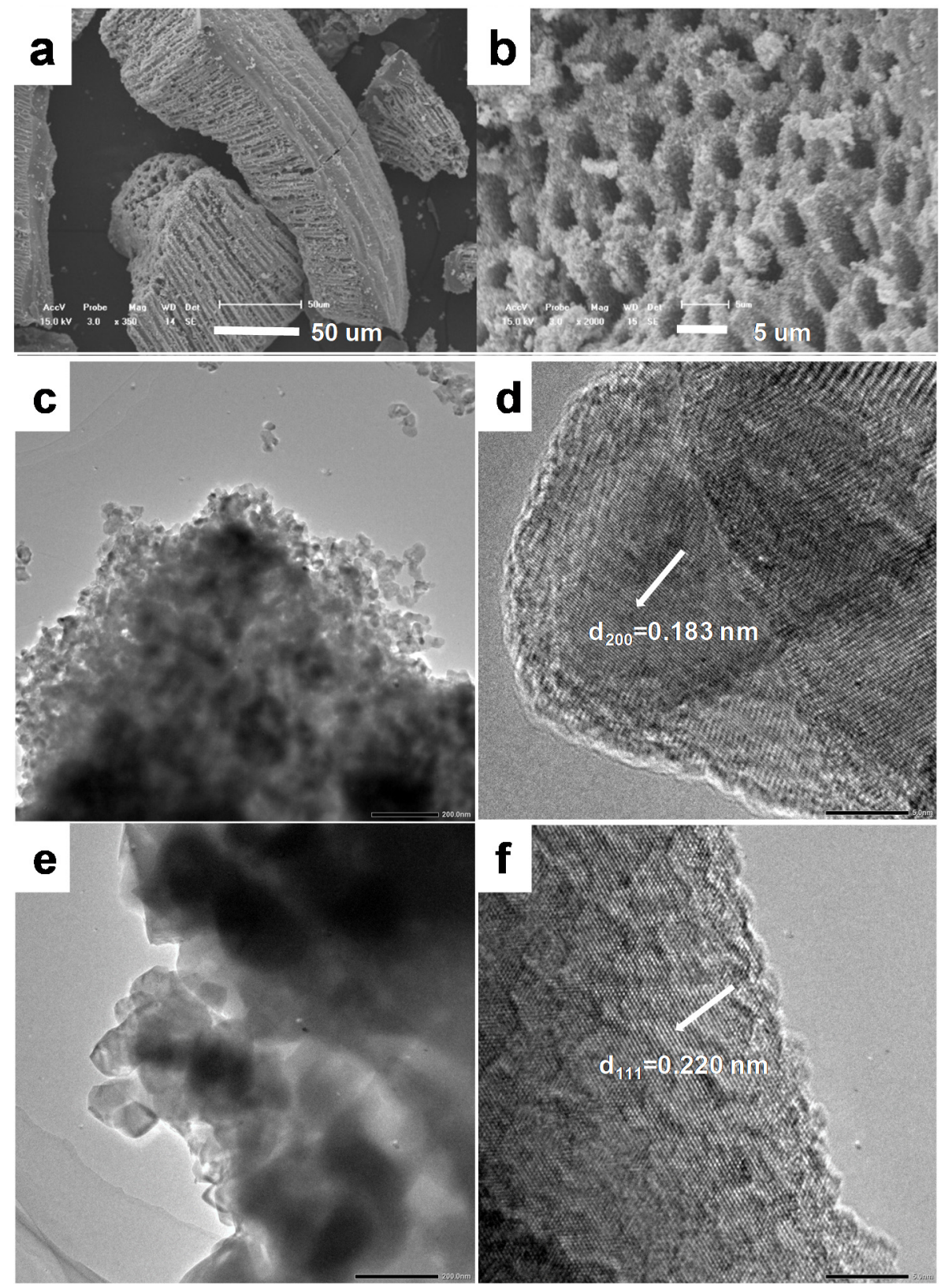

Figure 3. (a,b) SEM images of titanium oxide obtained by thermal decomposition at $800{ }^{\circ} \mathrm{C} \mathrm{TiO}_{2}-800$. (c,d) TEM imaged of $\mathrm{TiO}_{2}-550$. (e,f) TEM imaged of $\mathrm{TiO}_{2}-800$.
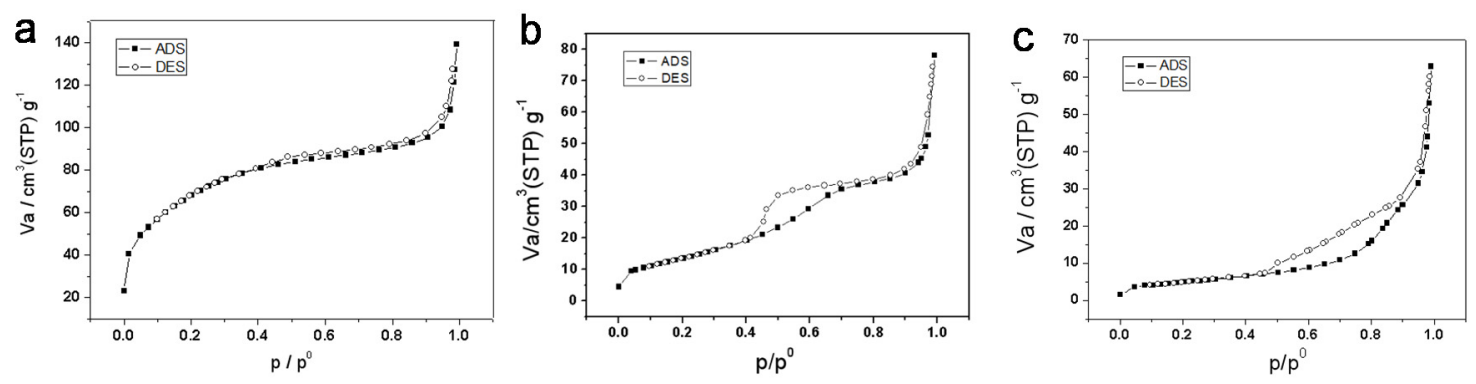

Figure 4. $\mathrm{N}_{2}$ adsorption-desorption curves of titanium samples, (a) titanium precursor, (b) $\mathrm{TiO}_{2}-550$, (c) $\mathrm{TiO}_{2}-800$. 


\subsection{Effect of Citric Acid on the Formation of Macroporous Structure}

In order to investigate the effect of citric acid on the formation of macroporous structure, the addition amount of citric acid was adjusted. When a certain amount of citric acid is added, the product forms a stable macroporous structure with a relatively high yield and a regular morphology (see Figure 5). When the amount of citric acid added to the reaction system increases to $0.72 \mathrm{~g}$ (Figure 5a,b), the closed end of the pores becomes extremely thin, many of the pores are converted into fully connected, and the dimeter of the macropores become more uniform. The arrangement of the macropore is more tight and tidy. When the amount of citric acid kept increasing to $1.8 \mathrm{~g}$ (Figure $5 \mathrm{c}, \mathrm{d}$ ), the yield of the macroporous structure was obviouly lowered, although neatly dense macropores were also retained. When the citric acid added to the reaction system was increased to $3.59 \mathrm{~g}$ (Figure $5 \mathrm{e}, \mathrm{f}$ ), almost no macroporous structure was found in the observation range, and instead some large pieces and blocks constructed by small particles were obtained.
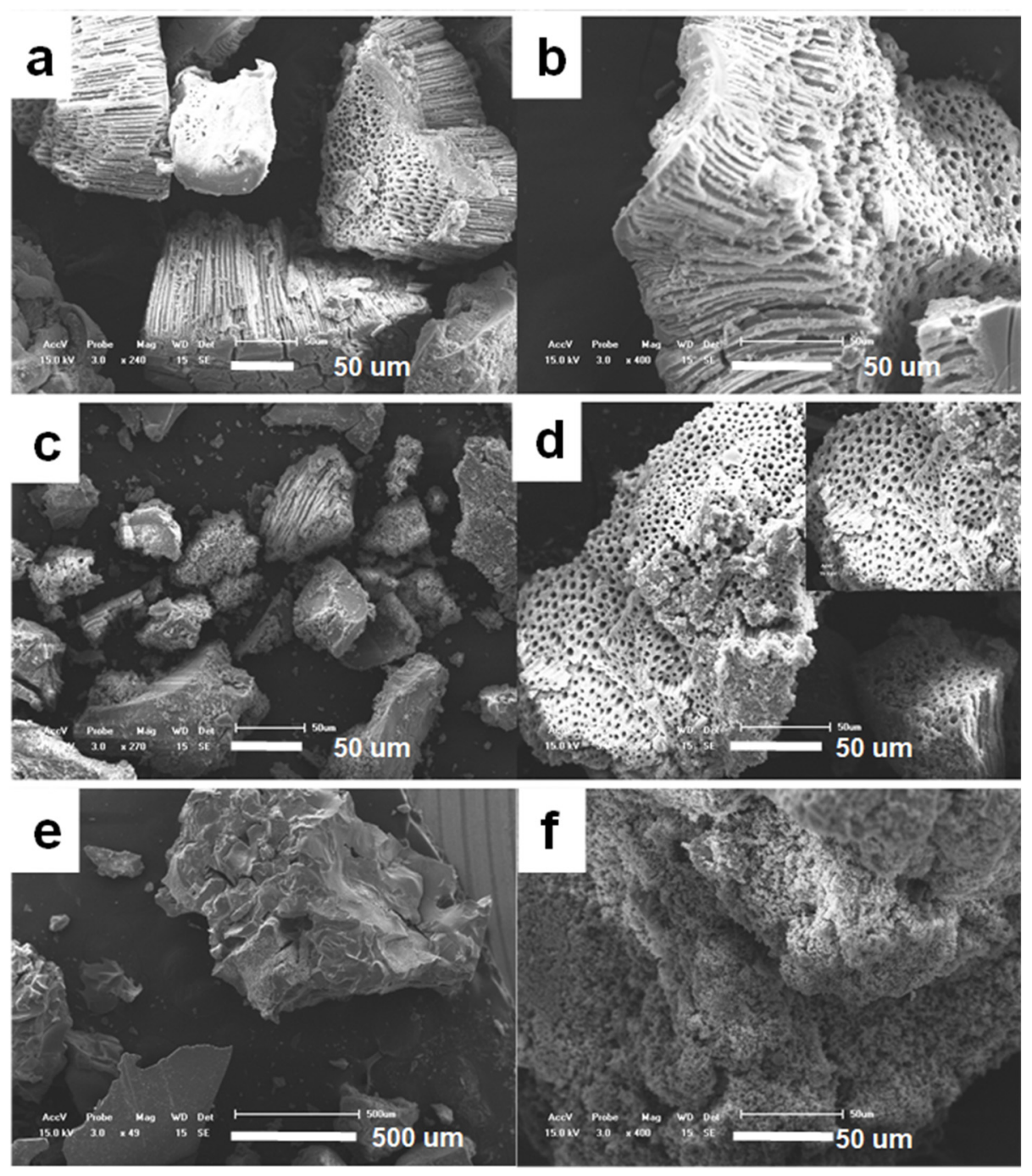

Figure 5. SEM images of titanium precursors prepared by the addition of citric acid. (a,b) $0.72 \mathrm{~g}$, (c,d) $1.8 \mathrm{~g},(\mathbf{e}, \mathbf{f}) 3.59 \mathrm{~g}$.

By adding different amounts of citric acid to the product, we can infer that the addition of a small amount of citric acid in the reaction system is beneficial to stabilize and optimize the macroporous structure of titanium dioxide, when the added citric acid is increased to about $0.72 \mathrm{~g}$ (i.e., the mole ratio of citric acid to titanium is 0.1 ), the large pore structure of titanium precursor with the highest mass yield is obtained. However, the mechanical stability of macroporous structure was poor. Further increasing 
the amount of citric acid causes the macroporous structure to gradually collapse. When the molar amount of citric acid is about 0.2 times that of the titanium source, the yield of macroporous titanium dioxide is significantly reduced; when the molar amount of citric acid reaches 0.5 times of the titanium source amount, no more macroprous structure can be observed in the product. This phenomenon may be caused by the presence of excess citric acid in the solution to destroy the conditions of macropore formation, such as $\mathrm{pH}$ value, or may be due to the large amount of ammonium citrate formed by the exceed addition of citric acid in the reaction system. The flocculent precipitate destroys the equilibrium of the reaction system and introduces more impurities.

Citric acid molecule is a ligand with multiple ligands, one hydroxyl group and three carboxyl groups. Citric acid (CA) has shown special abilities for creating mesoporous materials as organic additives in the sol-gel process [25-28]. For example, Liu Gang obtained amorphous mesoporous aluminophosphate materials (AlPO) with relatively high specific surface areas, narrow pore size distributions and excellent thermally stabilities. This method can be further extended to fabricate metal oxide. Porous nanometer nickel oxides with high surface area was fabricated through a CA involved sol-gel way. Thermally stable mesoporous anatase-nanocrystal framework was reported by a citric acid assisted templating method.

In our report, if citric acid is added to the precursor solution of titanium dioxide, citric acid will form a certain degree of complexation with titanium, so that the titanium dioxide precursor is highly dispersed in the organic ligand network. That is to say, the addition of citric acid would change the transition history of the precursor to the oxide and avoids the occurrence of agglomeration. Although we have no more understanding on the dynamics of various citrate microstructures, physicochemical properties, and thermal decomposition microscopic processes, the above experiments have confirmed the good coordination effect of citric acid, avoiding the formation of large agglomerated particles by the polycondensation reaction of hydrates during the formation of titanium macroporous precursors, which plays a very good role in stabilizing the macroporous framework.

\subsection{Photocatlytic Activity of Macroporous $\mathrm{TiO}_{2}$ with Different Crystalline Strcuture, (a) P25, (b) $\mathrm{TiO}_{2}-550$, (c) $\mathrm{TiO}_{2}-800$}

From the UV-visible diffuse reflection absorption spectrum, it can be clearly observed that P25 showed strong absorption in the UV region with an absorption edge at around $400 \mathrm{~nm}$. The absorption edge of $\mathrm{TiO}_{2}-550$ and $\mathrm{TiO}_{2}-800$ were slightly red-shifted. For $\mathrm{TiO}_{2}-550$, the difference could be ascribed to the lower amount of nitrogen incorporated, as revealed by EDS spectra (Figure 6c). For $\mathrm{TiO}_{2}-800$, the difference could be ascribed to two reasons. The first one is the different crystal phase, where $\mathrm{TiO}_{2}-800$ was a rutile $\mathrm{TiO}_{2}$, rutile phase usually shows a smaller band gap than anatase phase. However, for $\mathrm{TiO}_{2}-800$, it is still a red-shifted compared to pure rutile phase, this can also be attributed to a nitrogen doping effect. EDS spectra confirmed that N1s also existed in $\mathrm{TiO}_{2}-800$ (Figure 6d). During the synthesis of $\mathrm{TiO}_{2}$, ammonia was not only used as a precipitant, but also can provide nitrogen resources to produce a nitrogen-doped $\mathrm{TiO}_{2}$. Simultaneously, since the size of $\mathrm{TiO}_{2}-550$ is much smaller than $\mathrm{TiO}_{2}-800$, the nano-effect could also contribute to the blue shift. The band gap value of the catalyst is calculated according to the UV-vis spectra. By calculation, the band gap value of the catalyst is calculated according to the UV-vis spectra. By calculation, the bandgap energy of P25 is about $3.15 \mathrm{eV}$, $\mathrm{TiO}_{2}-550$ is about $2.93 \mathrm{eV}$, while that of $\mathrm{TiO}_{2}-800$ is about $2.73 \mathrm{eV}$.

As a typical water pollution agent, RB has been widely used in the photocatalytic reaction to evaluate the activity of photocatalyst. The photocatalytic test was performed under ultraviolet light irradiation with/without photocatalysts. In the blank control experiment, without any catalyst in the RB solution, the degradation of RB is slow as the reaction time increases. Only $14.3 \%$ of RB was degraded after a 20 min explosion of UV light. For macroporous $\mathrm{TiO}_{2}-550$, the conversion of $\mathrm{RB}$ reached $16.4 \%$ at the first 20 minutes' irradiation. The photocatalytic activity in the first $20 \mathrm{~min}$ for $\mathrm{TiO}_{2}-550$ is almost the same as that of the blank control, but it is quite different from the other catalysts. As a comparison, the photocatalytic activity of P25 under same condition was also investigated. As is 
shown in Figure 7, $\mathrm{TiO}_{2}-800$ exhibits a much higher photocatalytic activity $(75 \%$ removal of $\mathrm{RB}$ in the first $20 \mathrm{~min}$ ), similar with that of P25. After $40 \mathrm{~min}$, the RBs catalyzed by P25 and macroporous rutile materials were substantially degraded.
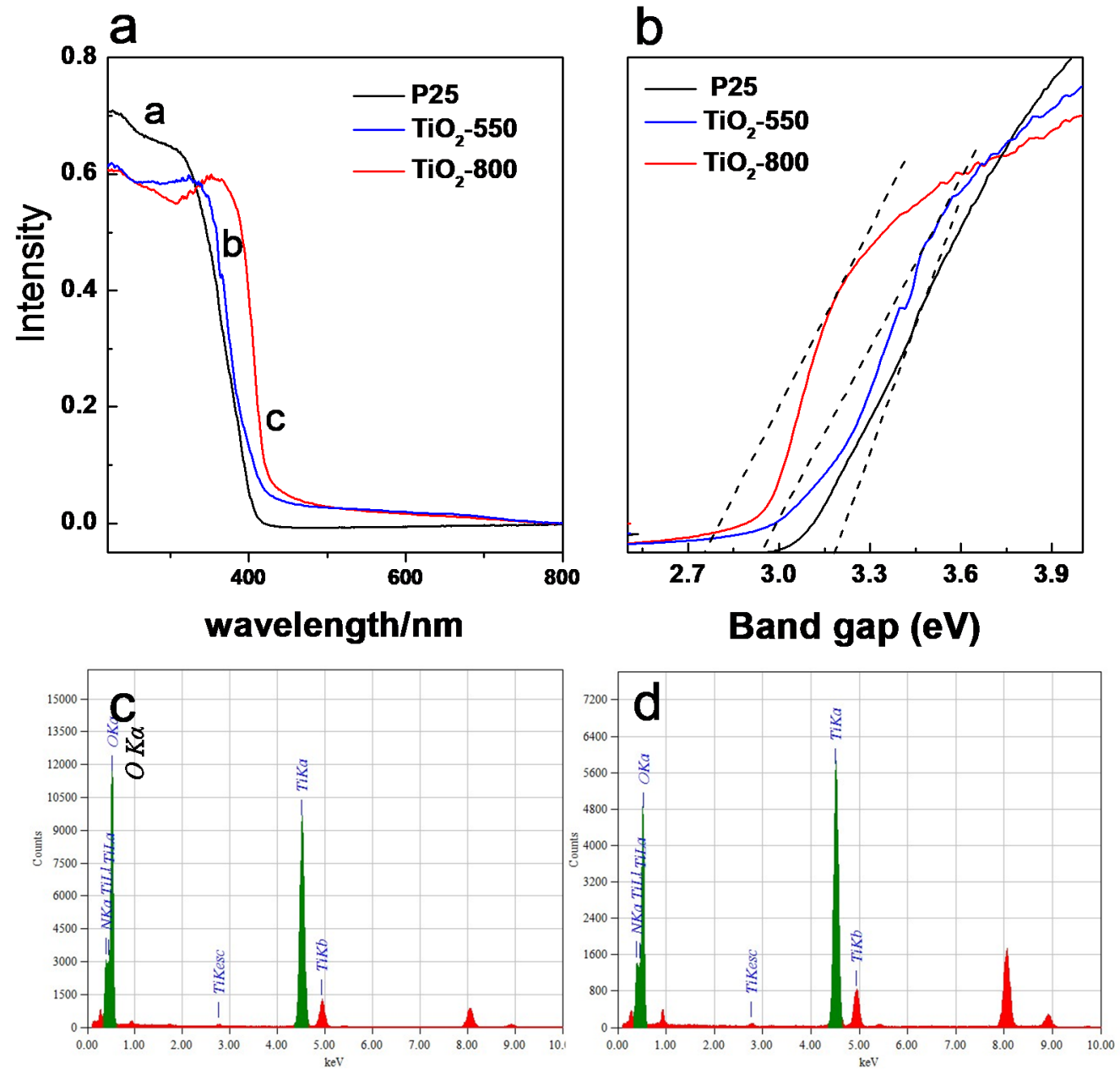

Figure 6. (a) UV-vis spectra of $\mathrm{TiO}_{2}$ samples, (b) calculated according to the formula $\alpha h v=\mathrm{A}(h v-\mathrm{Eg})^{\mathrm{n} / 2}$, (c,d) EDS spectra for $\mathrm{TiO}_{2}-550$ and $\mathrm{TiO}_{2}-800$.
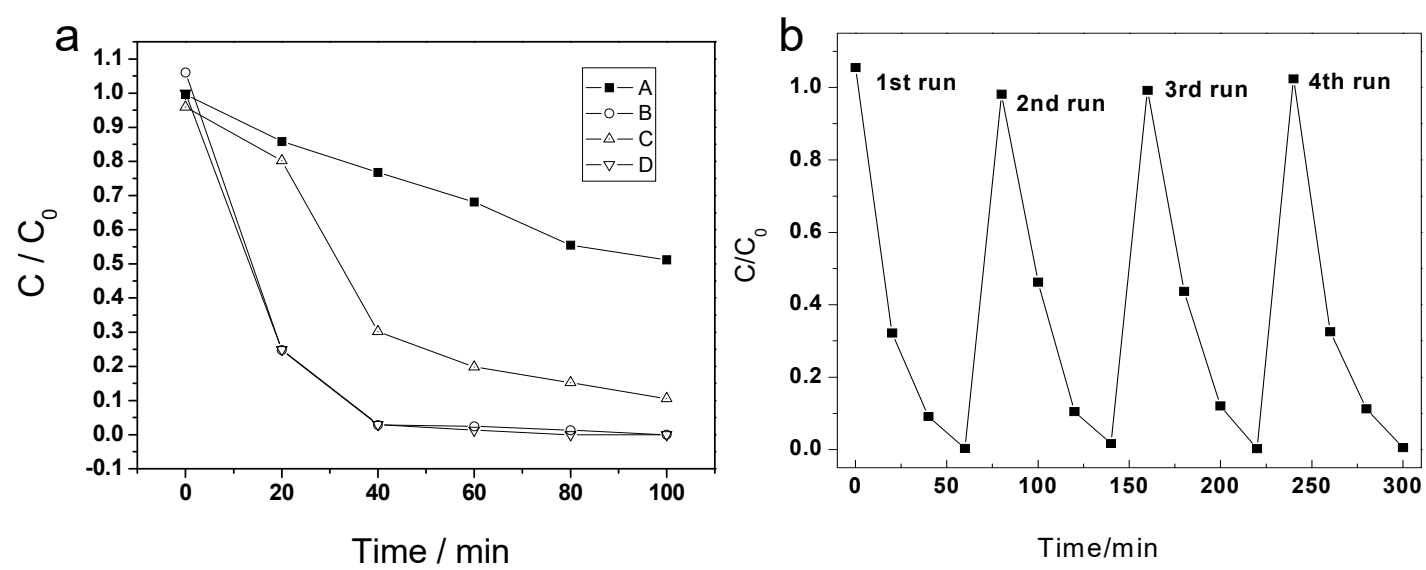

Figure 7. (a) Photocatalytic degradation of RB under UV-light irradiation, A-blank control, B-P25, $\mathrm{C}-\mathrm{TiO}_{2}-550, \mathrm{D}-\mathrm{TiO}_{2}-800$. (b) Recycling test for $\mathrm{RB}$ degradation over $\mathrm{TiO}_{2}-800$. 
Since the $\mathrm{N}_{2}$ adsorption results have been shown to have been destroyed by high temperature calcination at $550{ }^{\circ} \mathrm{C}$ and $800{ }^{\circ} \mathrm{C}$, the micropores structures accumulated in the titanium dioxide material have been destroyed, so there is basically no existence of micropores. The product contains large macropores with a pore size of several micrometers visible in SEM, and some mesopores existed between the nanoparticles aggregation, as observed in TEM. The adsorption of the dye molecules by the photocatalyst could be ignored, it can be inferred that decolorization reaction of the dye solution is the result of photocatalytic degradation.

The photocatalytic activity mainly depends on three aspects: the absorption rate of light, the reaction efficiency of photogenerated electron/hole pairs, and the recombination rate of electron/hole pairs. An important factor affecting the light absorption rate is the pore wall structure of the catalyst. Ultraviolet light decays rapidly with its propagation in the medium. Ultraviolet light with a wavelength of $320 \mathrm{~nm}$ is propelled by $8.5 \mathrm{um}$ in titanium dioxide solids, and its intensity is attenuated to $10 \%$ of the original intensity. In this experiment, the cylindrical straight channel of macroporous titania provides an optical path for the photon stream to enter the inner surface of the catalyst. The macroporous structure causes light waves to be injected into the inner layer of the catalyst, which enhances the light absorption efficiency and greatly improves the catalytic efficiency of the photocatalyst.

According to the XPS results (see Figure 8), the binding energies of Ti $2 \mathrm{p}_{3 / 2}$ and Ti $2 \mathrm{p}_{1 / 2}$ are $458.4 \mathrm{eV}$ and $464.1 \mathrm{eV}$, respectively. The binding energy of $\mathrm{O} 1 \mathrm{~s}$ is $529.6 \mathrm{eV}$. There was a single peak in $\mathrm{N} 1 \mathrm{~s}$ spectrum for $\mathrm{TiO}_{2}-800,399.4 \mathrm{eV}$, which is greater than the binding energy of $\mathrm{N} 1 \mathrm{~s}$ in TiN and smaller than the binding energy of $\mathrm{N} 1 \mathrm{~s}$ in $\mathrm{NaNO}_{3}$. N1s binding energy at $399.4 \mathrm{eV}$ is $0.6 \mathrm{eV}$ higher from the standard binding energy of $\mathrm{N} 1 \mathrm{~s}$ in $\mathrm{NH}_{3}(398.8 \mathrm{eV})$, which is the only nitrogen source in this experiment. Due to the existence of a large amount of oxygen with higher electronegativity than nitrogen in the crystal lattice of $\mathrm{TiO}_{2}-800$, the electron density decreases and the binding energy of N1s increases. It can be concluded that nitrogen atom in the product may exist as O-Ti-N. Numerous efforts have been devoted to achieving higher separation efficiency through nanostructure engineering, doping strategy, surface treatment, and junction-structure modification [28-30]. In this report, ammonia was used as a doping element to get a $\mathrm{N}$-doped $\mathrm{TiO}_{2}$, which can extend the photoresponsed range and further enhance the photocatalytic activity. The experimental results demonstrated that this modified nitrogen doped rutile $\mathrm{TiO}_{2}$ sample exhibits enhanced absorption in visible region and may induce efficient charge separation and transport. As a result, the unique nitrogen surface $\left(\mathrm{TiO}_{2-x}: \mathrm{N}\right)$ enhance the photocatalytic RhB degradation.
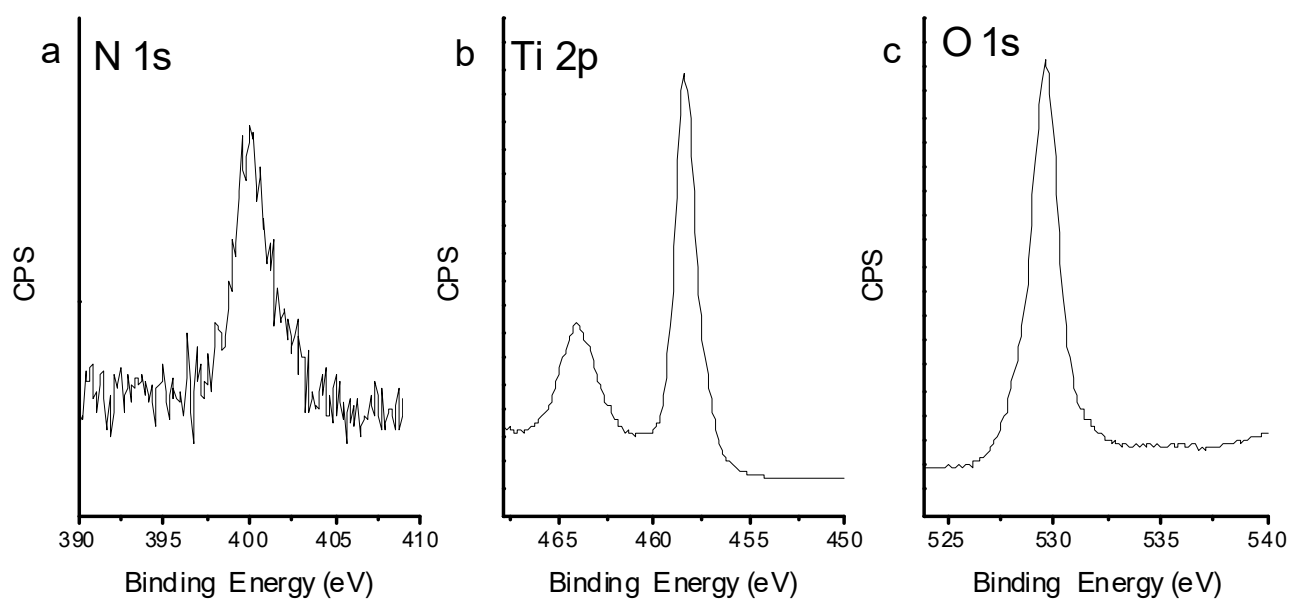

Figure 8. XPS spectra of $\mathrm{TiO}_{2}-800$, (a) N 1s spectrum, (b) Ti 2p spectrum, (c) O 1s spectrum.

\section{Conclusions}

We reported a citric stabilized preparation of microporous-macroporous titanium dioxide. The experimental results show that the product can hardly form regular macroporous channels 
without the addition of citric acid. Under the same reaction conditions, when certain amount of citric acid was added, the titanium precursor exhibits a large range of macroporous structure. When the amount of citric acid added is about $0.36 \mathrm{~g}$, the yield of a stable macroporous structure is the highest. After calcination at different temperatures, nitrogen-doped macroporous titanium oxide of rutile and anatase phases were obtained. Compared with P25, both of the rutile and the anatase phase macroporous $\mathrm{TiO}_{2}$ showed good catalytic activity for photocatalytic degradation of RB.

Author Contributions: X.Z., R.C. and N.S. performed the experiments. R.C. Wrote the manuscript. T.C. took part in data analysis. All authors have read and agreed to the published version of the manuscript.

Funding: This work was supported by NSFC (no. 21373116) of China.

Conflicts of Interest: The authors declare no conflict of interest.

\section{References}

1. Chen, X.B.; Burda, C. The Electronic Origin of the Visible-Light Absorption Properties of C-, N- and S-Doped $\mathrm{TiO}_{2}$ Nanomaterials. J. Am. Chem. Soc. 2008, 130, 5018-5019. [CrossRef] [PubMed]

2. Chen, X.B.; Liu, L.; Huang, F.Q. Black Titanium Dioxide $\left(\mathrm{TiO}_{2}\right)$ Nanomaterials. Chem. Soc. Rev. 2015, 44, 1861-1885. [CrossRef] [PubMed]

3. Thompson, T.L.; Yates, J.T. Surface Science Studies of the Photoactivation of $\mathrm{TiO}_{2}$ New Photochemical Processes. Chem. Rev. 2006, 106, 4428-4453. [CrossRef] [PubMed]

4. Zhang, J.; Xu, Q.; Feng, Z.C.; Li, M.J.; Li, C. Importance of the Relationship Between Surface Phases and Photocatalytic Activity of $\mathrm{TiO}_{2}$. Angew. Chem. Int. Ed. 2008, 47, 1766-1769. [CrossRef]

5. Gutierrez, J.; Tercjak, A.; Mondragon, I. Conductive Behavior of High $\mathrm{TiO}_{2}$ Nanoparticle Content of Inorganic/Organic Nanostructured Composites. J. Am. Chem. Soc. 2010, 132, 873-878. [CrossRef]

6. Kwon, D.H.; Kim, K.M.; Jang, J.H.; Jeon, J.M.; Lee, M.H.; Kim, G.H.; Li, X.S.; Park, G.S.; Lee, B.; Han, S.; et al. Atomic Structure of Conducting Nanofilaments in $\mathrm{TiO}_{2}$ Resistive Switching Memory. Nat. Nanotechnol. 2010, 5, 148-153. [CrossRef]

7. Bavykin, D.V.; Friedrich, J.M.; Walsh, F.C. Protonated Titanates and $\mathrm{TiO}_{2}$ Nanostructured Materials: Synthesis, Properties, and Applications. Adv. Mater. 2006, 18, 2807-2824. [CrossRef]

8. Fujishima, A.; Zhang, X.; Tryk, D. TiO 2 Photocatalysis and Related Surface Phenomena. Surf. Sci. Rep. 2008, 63, 515-582. [CrossRef]

9. Khan, M.M.; Ansari, S.A.; Pradhan, D.; Ansari, M.O.; Han, D.H.; Lee, J.; Cho, M.H. Band Gap Engineered $\mathrm{TiO}_{2}$ Nanoparticles for Visible Light Induced Photoelectrochemical and Photocatalytic Studies. J. Mater. Chem. A 2014, 2, 637-644. [CrossRef]

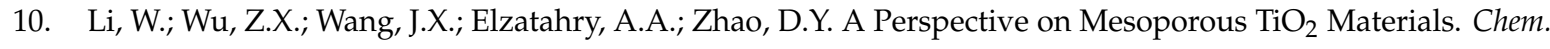
Mater. 2014, 26, 287-298. [CrossRef]

11. Schneider, J.; Matsuoka, M.; Takeuchi, M.; Zhang, J.; Horiuchi, Y.; Anpo, M.; Bahnemann, D.W. Understanding $\mathrm{TiO}_{2}$ Photocatalysis: Mechanisms and Materials. Chem. Rev. 2014, 114, 9919-9986. [CrossRef] [PubMed]

12. Tian, J.; Zhao, Z.H.; Kumar, A.; Boughton, R.I.; Liu, H. Recent Progress in Design, Synthesis, and Applications of One-dimensional $\mathrm{TiO}_{2}$ Nanostructured Surface Heterostructures: A review. Chem. Soc. Rev. 2014, 43, 6920-6937. [CrossRef] [PubMed]

13. Yuan, Z.Y.; Ren, T.Z.; Azioune, A.; Pireaux, J.J.; Su, B.L. Self-Assembly of Hierarchically Mesoporous-Macroporous Phosphated Nanocrystalline Aluminum (Oxyhydr)oxide Materials. Chem. Mater. 2006, 18, 1753-1767. [CrossRef]

14. Shi, M.M.; Bao, D.; Wulan, B.R.; Li, Y.H.; Zhang, Y.F.; Yan, J.M.; Jiang, Q. Au Sub-Nanoclusters on TiO 2 toward Highly Efficient and Selective Electrocatalyst for $\mathrm{N}_{2}$ Conversion to $\mathrm{NH}_{3}$ at Ambient Conditions. Adv. Mater. 2017, 29, 1606550. [CrossRef] [PubMed]

15. Choi, H.; Sofranko, A.C.; Dionysiou, D.D. Nanocrystalline $\mathrm{TiO}_{2}$ Photocatalytic Membranes with a Hierarchical Mesoporous Multilayer Structure: Synthesis, Characterization, and Multifunction. Adv. Funct. Mater. 2006, 16, 1067-1074. [CrossRef]

16. Kandiel, T.A.; Dillert, R.; Feldhoff, A.; Bahnemann, D.W. Direct Synthesis of Photocatalytically Active Rutile $\mathrm{TiO}_{2}$ Nanorods Partly Decorated with Anatase Nanoparticles. J. Phys. Chem. C 2010, 114, 4909-4915. [CrossRef] 
17. Kim, S.H.; Cho, Y.S.; Jeon, S.J.; Eun, T.H.; Yi, G.R.; Yang, S.M. Microspheres with Tunable Refractive Index by Controlled Assembly of Nanoparticles. Adv. Mater. 2008, 20, 3268-3273. [CrossRef]

18. Li, H.X.; Bian, Z.F.; Zhu, J.; Zhang, D.Q.; Li, G.S.; Huo, Y.N.; Li, H.; Lu, Y.F. Mesoporous Titania Spheres with Tunable Chamber Stucture and Enhanced Photocatalytic Activity. J. Am. Chem. Soc. 2007, 129, 8406-8407. [CrossRef]

19. Song, X.F.; Gao, L. Fabrication of Hollow Hybrid Microspheres Coated with Silica/Titania via Sol-Gel Process and Enhanced Photocatalytic Activities. J. Phys. Chem. C 2007, 111, 8180-8187. [CrossRef]

20. Wang, M.Y.; Wang, C.L.; Xie, K.P.; Sun, L.; Lin, C.J. Fabrication of a Sponge like Nanostructured $\mathrm{TiO}_{2}$ Film and Its Photocatalytic Activity. Acta Phys. Chim. Sin. 2009, 25, 2475-2480.

21. Xu, P.C.; Liu, Y.; Wei, J.H.; Xiong, R.; Pan, C.X.; Shi, J. Solvothermal Preparation of Ag/TiO 2 Nanoparticles and Their Photocatalytic Activity. Acta Phys. Chim. Sin 2010, 26, 2261-2266.

22. Yu, J.G.; Su, Y.R.; Cheng, B. Template-Free Fabrication and Enhanced Photocatalytic Activity of Hierarchical Macro-/Mesoporous Titania. Adv. Funct. Mater. 2007, 17, 1984-1990. [CrossRef]

23. Wu, Q.Z.; Shen, Y.; Liao, J.F.; Li, Y.G. Synthesis and Characterization of Three-dimensionally Ordered Macroporous Rare Earth Oxides. Mater. Lett. 2004, 58, 2688-2691. [CrossRef]

24. Song, L.H.; Li, L.; Gao, X.; Zhao, J.X.; Lu, T.; Liu, Z. A Facile Synthesis of a Uniform Constitution of Three-Dimensionally Ordered Macroporous $\mathrm{TiO}_{2}$-carbon Nanocomposites with Hierarchical Pores for Lithium Ion Batteries. J. Mater. Chem. A 2015, 3, 6862-6872. [CrossRef]

25. Fan, Y.; Bao, X.J.; Lin, X.Y.; Shi, G.; Liu, H.Y. Acidity Adjustment of HZSM-5 Zeolites by Dealumination and Realumination with Steaming and Citric Acid Treatments. J. Phys. Chem. B 2006, 110, 15411-15416. [CrossRef]

26. Shen, X.Q.; Zhou, J.X.; Jing, M.X.; Shen, Y.J. Nanosized Nickel Oxides Derived from the Citrate Gel Process and Performances for Electrochemical Capacitors. J. Wuhan Univ. Technol. Mater. Sci. Ed. 2007, 22, 179-182. [CrossRef]

27. Liu, G.; Jia, M.J.; Zhou, Z.; Zhang, W.X.; Wu, T.H.; Jiang, D.Z. Synthesis of Amorphous Mesoporous Aluminophosphate Materials with High Thermal Stability Using a Citric Acid Route. Chem. Commun. 2004, 1660. [CrossRef]

28. Delekar, S.; Yadav, H.; Achary, S.N.; Meena, S.S.; Pawar, S. Structural Refinement and Photocatalytic Activity of Fe-doped Anatase $\mathrm{TiO}_{2}$ Nanoparticles. Appl. Surf. Sci. 2012, 263, 536-545. [CrossRef]

29. Yadav, H.M.; Otari, S.V.; Koli, V.B.; Mali, S.S.; Hong, C.K.; Pawar, S.H.; Delekar, S.D. Preparation and Characterization of Copper-doped Anatase $\mathrm{TiO}_{2}$ Nanoparticles with Visible Light Photocatalytic Antibacterial Activity. J. Photochem. Photobiol. A Chem. 2014, 280, 32-38. [CrossRef]

30. Gao, C.M.; Wei, T.; Zhang, Y.Y.; Song, X.H.; Huan, Y.; Liu, H.; Zhao, M.W.; Yu, J.H.; Chen, X.D. A Photoresponsive Rutile $\mathrm{TiO}_{2}$ Heterojunction with Enhanced Electron-Hole Separation for High-Performance Hydrogen Evolution. Adv. Mater. 2019, 31, 1806596. [CrossRef] 